\title{
Desvios axiais dos membros inferiores
}

M. Cassiano Neves, ${ }^{*}$ João Lameiras Campagnolo**

\section{RESUMO}

As deformidades axiais dos membros inferiores constituem um dos principais motivos de consulta na área de Ortopedia Infantil. Esta situação deve-se, por um lado, ao facto de haver uma grande percentagem de crianças (cerca de $20 \%$ ) que apresentam uma deformidade fisiológica durante o desenvolvimento e, por outro, à «tradição» existente, segundo a qual estes desvios necessitam de um tratamento, usualmente por calçado ortopédico.

Torna-se, por isso, imperioso destrinçar o fisiológico do patológico, diferenciar as diferentes etiologias e corrigi-las precocemente medicamente e, ainda, determinar quando uma deformidade é passível de correcção espontânea ou quando exige uma terapêutica cirúrgica.

Palavras-chave: Desenvolvimento; Joelhos Varos; Joelhos Valgus; Recurvato.

\section{INTRODUÇÃO}

A s deformidades dos membros inferiores no plano frontal são uma das causas mais frequentes de consulta em Ortopedia, quer a pedido dos pais, quer dos médicos assistentes.

As deformidades axiais à nascença são muito raras e estão, na maioria dos casos, subjacentes a anomalias congénitas dos ossos longos, com graves repercussões no desenvolvimento do membro. A excepção será a tíbia em «S», que consiste numa deformidade axial da tíbia, com uma curvatura com vértice posterior e interno dando, à nascença, a posição de um "pé talo» muito acentuado. Esta deformidade é considerada «benigna" porque corrige espontaneamente com o desenvolvimento, apresentando como única sequela na idade adulta um encurtamento da perna entre os 2 e os $4 \mathrm{~cm} .^{1}$

Na verdade, as modificações do alinhamento dos membros inferiores durante os primeiros seis anos de vida são, na sua grande maioria, fisiológicas, pelo que apenas uma escassa minoria dos casos necessitam de tratamento ortopédico.

Antes do mais, deveremos distinguir as deformações dos joelhos em varo (joelhos afastados com os torno-

*Chefe de Serviço; Responsável da Unidade de Ortopedia. Departamento Cirurgia Pediátrica - Hospital Dona Estefânia *Assistente Hospitalar. Unidade de Ortopedia, Departamento Cirurgia Pediátrica Hospital Dona Estefânia zelos em contacto), das deformações em valgo (os joelhos aproximam-se da linha média e as pernas ficam divergentes, com um exagerado afastamento dos tornozelos). Seguidamente, em função da idade da criança, da gravidade da deformidade, dos antecedentes pessoais e familiares e da existência ou não de patologia sistémica relacionada, tomaremos uma eventual decisão terapêutica.

É de salientar que, no final do crescimento, o morfotipo em varo dos joelhos é mais frequente no homem (60\%) que na mulher (40\%), enquanto que o morfotipo em valgo é mais frequente no sexo feminino.

\section{FISIOPATOLOGIA}

Os mecanismos que conduzem a desvios axiais dos membros inferiores no plano frontal são diferentes consoante o tipo de deformidade, em valgo ou em varo. De qualquer modo, e em qualquer dos casos, distinguem-se os seguintes mecanismos:

- Fisiológico

- Não fisiológico: idiopático secundário

- Fisiológico: na sua posição intra-uterina, as crianças encontram-se com os membros sobrepostos e enrolados um sobre o outro, o que condiciona uma deformidade em varo dos membros inferiores, associada a uma rotação interna das tíbias e a uma ante- 


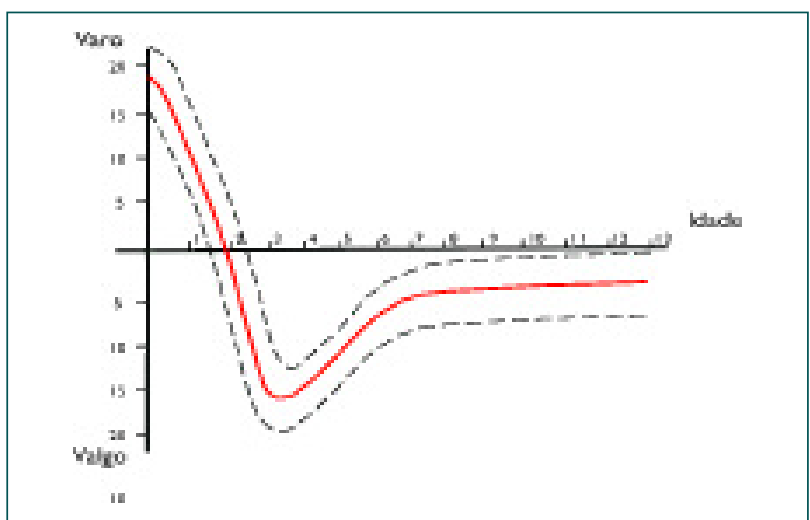

Figura 1. Evolução do eixo mecânico dos membros inferiores durante o crescimento

versão dos colos do fémur. Nos dois primeiros anos, fruto da acção muscular, estas deformidades vão corrigindo espontaneamente, ajudadas pelo hiper-crescimento do côndilo femoral interno, o que acontece cerca dos 2 anos de idade.

É, por isso, frequente observar um varismo entre o $1^{\circ} \mathrm{e} 2^{\circ}$ ano de vida e um valgismo entre o $2^{\circ}$ e $6^{\circ}$ ano de vida conforme o quadro anexo (Figura 1). É uma situação geralmente assintomática, simétrica, benigna, de resolução espontânea, que não precisa de tratamento.

- Não fisiológico: resulta de alterações biomecânicas mantidas e progressivamente agravadas, que conduzem a um desequilíbrio das forças exercidas a nível do joelho, com sobrecarga, seja do compartimento interno (varo), seja do externo (valgo).

\section{A) Geno varo}

Idiopático: observam-se casos de geno varo persistente após o $2^{\circ}$ ano de vida, com angulação significativa, mas que corrigem espontaneamente após 1-2 anos de evolução. Nestes casos as epífises são radiologicamente normais.

Secundário é mais frequente dos 3 aos 10 anos e com causas semelhantes às do geno valgo secundário, mas com alterações biomecânicas que afectam preponderantemente o compartimento interno do joelho. Pode ser causado por:

Doenças de desenvolvimento, das quais a mais frequente é a Doença de Blount

- Doenças metabólicas: raquitismo

- Doenças genéticas: displasias ósseas
- Doenças reumatológicas: ex. artrite inflamatória

- Traumatismos: epifisiodeses assimétricas

- Infecções: poliomielite

- Outras causas

\section{B) Geno valgo}

Idiopático: é mais frequente em adolescentes; coexiste, geralmente, com dor anterior e interna do joelho, associada a uma marcha com necessidade de um joelho «contornar» o outro (marcha circundante) e, por vezes, com instabilidade patelo-femoral.

Trata-se de uma situação habitualmente progressiva, que não corrige espontaneamente e que requer eventual tratamento cirúrgico.

Secundário: é mais comum dos 3 aos 10 anos e as causas mais frequentemente associadas são:

- Doenças metabólicas: raquitismo

- Doenças genéticas: displasias ósseas, Síndroma de Down, neurofibromatose, Síndroma de Marfan

- Doenças reumatológicas: ex. artrite inflamatória

- Traumatismos: ex. fractura proximal da tíbia*

- Infecções: poliomielite

- Outras causas: ex. hemofilia

\section{SEMIOLOGIA CLÍNICA}

$\mathrm{Na}$ avaliação dos desvios de eixo, existem dados que se devem particularmente evidenciar:

\section{História clínica:}

- Motivo da consulta, história natural das queixas/ /deformidades, início das queixas/deformidades e sua progressão.

Refira-se que os desvios de eixo são, habitualmente, assintomáticos, sendo de salientar que as deformidades assimétricas devem ser sempre valorizadas, pois traduzem a existência de patologia subjacente. Do mesmo modo, também a progressão de um desvio deve ser sempre alvo de atenção: um varismo que progride depois dos 2 anos não é, com certeza, fisiológico, o mesmo se passando com um valgismo que progride depois dos 4 anos.

\footnotetext{
* Caso particular do geno valgo pós-traumático: fractura da metáfise proximal da tíbia com ou sem fractura do peróneo ("fractura de Cozen"); neste caso terá um tratamento com gesso cruro-podálico em ligeiro varo do joelho; a máxima deformidade em geno valgo será evidente depois de um ano e a resolução, espontânea, pode demorar vários anos, sendo controversa a necessidade de cirurgia. ${ }^{2}$
} 


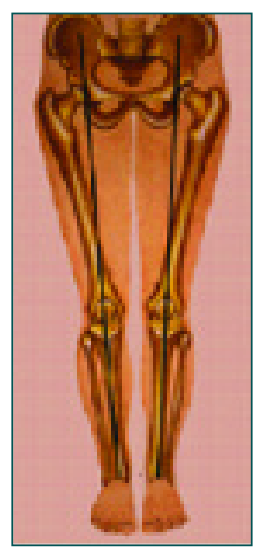

Figura 2. Eixo mecânico

- Antecedentes pessoais: dieta, doenças prévias, antecedentes traumáticos

- Antecedentes familiares: factor hereditário

\section{Exame objectivo:}

Um exame clínico sucinto informa acerca do morfótipo da criança: o eixo anatómico de carga do membro inferior passa, normalmente, pelas espinhas das tíbias; no caso de um joelho valgo, passa por fora e, num joelho varo, por dentro. ${ }^{3}$

A observação deve ser efectuada com as rótulas ao zénite e uma rotação dos pés para pôr a rótula ao zénite.

Devem, ainda, valorizar-se os seguintes dados:

- Peso e altura, pois podem agravar as condições mecânicas do eixo de carga

- Deformidade localizada/generalizada

- Dismetrias dos membros inferiores

- Hiperlaxidão ligamentar: geno valgo que aumenta em carga

- Joelhos que «roçam na marcha» $\rightarrow$ Marcha em «circundução»

- Gonalgia no compartimento medial e anterior em carga

- Casos mais graves: luxação da rótula e fracturas osteo-condrais

- Avaliação dos desalinhamentos rotacionais dos membros inferiores:

- Aumento da anteversão femoral

- Rotação tibial interna (pseudo geno valgo)

- Pé plano valgo

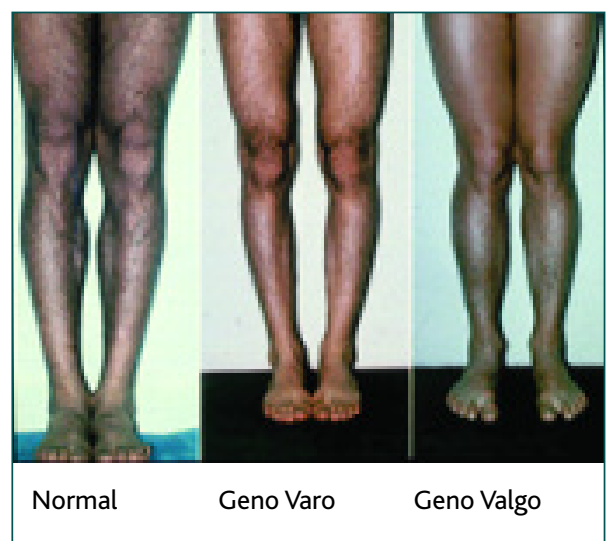

Figura 3. Desvios de Eixo (plano frontal)

- Rotação externa da tíbia compensada com o geno valgo

(Nota: o geno valgo patológico não corrige com as rótulas ao zénite)

A deformidade é avaliada pela distância inter-maleolar (DIM - o valor normal é inferior a $2 \mathrm{~cm}$ ), quando os joelhos estão em contacto. Pode ser sobre-avaliada em indivíduos obesos com coxas volumosas, em que os côndilos femorais não se conseguem encostar (nestes casos, quando uma indicação cirúrgica é ponderada, a avaliação deve ser radiológica). ${ }^{5}$

\section{Factores associados a cada tipo de desvio}

\section{A) Geno varo}

Habitualmente, crianças com cerca de 2-3 anos e com:

- Raquitismo

- Doença de Blount: associado ao início precoce da marcha é a obesidade. Neste caso particular consegue-se palpar um «bico» ósseo no lado interno, correspondente à proeminência da metáfise proximal da tíbia.

\section{B) Geno valgo}

Frequentemente, crianças com cerca de 4-6 anos e com:

- Baixa estatura

- Traumatismo prévio

- História de infecção

- Doença metabólica

- Assimetria importante, sugerindo situação patológica 


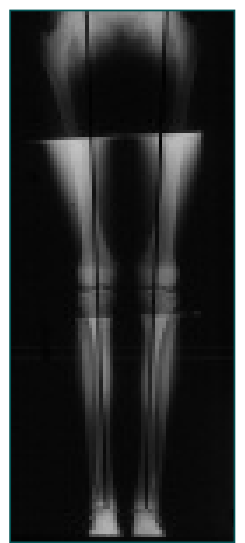

Figura 4. Eixo mecânico

\section{EXAMES COMPLEMENTARES DE DIAGNÓSTICO}

\section{A) Imagiologia}

O diagnóstico de uma deformidade patológica deve ser imagiológico.

Os exames a pedir serão:

- Radiografia (RX) extra-longo em carga dos membros inferiores

- RX dos joelhos nos 2 planos (antero posterior (AP) + perfil, para definição de eventuais alterações locais) Poderão ser analisados:

- 1 . O eixo mecânico (divide o joelho em 2 partes iguais)

- 2. O ângulo femoro-tibial

- 3. Outras medições:

- Ângulo femoral lateral distal $\left(\mathrm{N}=94^{\circ}\right.$, valgo $\left.=6^{\circ}\right)$

- Ângulo proximal medial tibial ( $\mathrm{N}=3^{\circ}$ Varo)

Acessoriamente, se houver suspeita de outras patologias associadas, poderão ser pedidos outros exames
Valores normais:

- Ângulo femoro-tibial

$\mathrm{N}=\sigma^{\circ} 6^{\circ}+8^{\circ}$

- deitado (rótula centrada)

- em carga

- DIM em carga

$\mathrm{N}<2 \mathrm{~cm}$

- Deitado (rótula centrada)

- Em carga

Figura 5. Valores referência (DIM e ângulo femoro-tibial) imagiológicos:

- RX coxo-femorais AP+P (se o RX extra-longo detectar anomalias da fise femoral proximal)

- RX do punho/mão em AP-lado não dominante, para determinação da idade óssea

- Outras incidências especiais

- Tomografia axial computorizada (TAC), ressonância magnética nuclear (RMN)

\section{B) Exames laboratoriais}

Perante uma deformidade generalizada, tem indicação o pedido de exames laboratoriais, em especial o estudo do metabolismo fosfo-cálcico, para rastreio de uma doença metabólica que possa estar subjacente (p.e., o raquitismo); nessas situações, o doente deve, também, ser referenciado para uma consulta de genética e endocrinologia.

\section{CLASSIFICAÇÃO}

\section{A) Geno varo}

Classificação de Langenskiöld da doença de Blount em 6 graus. Trata-se de uma classificação radiográfica que nos indica o prognóstico. A partir do grau III existem repercussões futuras em termos de crescimento, ${ }^{6}$ pelo que esta doença obriga a um tratamento precoce.

B) Geno valgo: distância intermaleolar normal $<2 \mathrm{~cm}$

GV LEVE $=2-5 \mathrm{~cm}$

MODERADO $=5-9 \mathrm{~cm}$

GRAVE $>9 \mathrm{~cm}$

DIAGNÓSTICO DIFERENCIAL

Geno valgo/varo

- Fisiológico

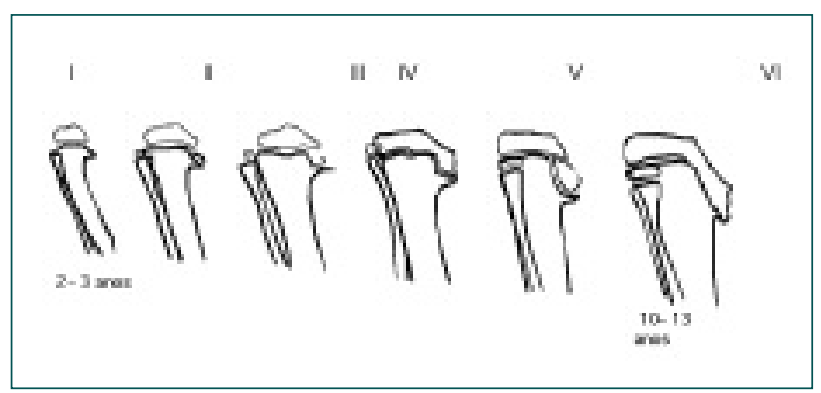

Figura 6. Classificação de Langenskiöld da Doença de Blount 
- Displasias esqueléticas (condrodisplasia metafisária, displasia espondilo-epifisária, displasia epifisária múltipla, acondroplasia)

- Doenças metabólicas (osteodistrofia renal, raquitismo resistente à vitamina $\mathrm{D}$ )

- Pós-traumático

- Pós-infeccioso

- Displasia fibro-cartilagínea focal

\section{Geno varo}

- Doença de Blount

\section{ATITUDE/PROCEDIMENTOS DO MÉDICO DE FAMÍLIA/PEDIATRA}

A primeira noção a ter em conta é que as deformidades axiais dos membros não se tratam com sapatos correctivos, vulgarmente chamados ortopédicos. Há 20 anos atrás, era comum receitar as ortóteses tipo «talas de sereia» para correcção destas alterações. Hoje em dia, é difícil convencer uma criança de 8 anos ou uma adolescente $\mathrm{a}$ «dormir de pernas atadas». Por isso, o princípio básico do tratamento destas deformidades é a correcção dos factores etiológicos que possam estar subjacentes e, perante a falência da terapêutica conservadora, a cirurgia.

O geno varo persistente após os 2 anos de idade é uma indicação para o envio à consulta de Ortopedia, pois uma deformidade evoluída pode ter repercussões graves na idade adulta.

Quanto ao geno valgo, é uma patologia frequente e benigna, mas deve ser enviada ao especialista quando se verifica a presença de:

- deformidades múltiplas associadas ao esqueleto

- deformidade progressiva a partir dos 4 anos

- geno valgo grave (mais de $9 \mathrm{~cm}$ de distância intermaleolar)

- geno valgo sintomático

- crianças entre os 6-8 anos com geno valgo sem resolução espontânea

- assimetria

\section{PERSPECTIVA DO PLANO TERAPÊUTICO DO CIRURGIÃO ORTOPÉDICO}

A orientação terapêutica tem indicações em função do tipo de geno valgo ou varo:

- Deformidades fisiológicas: não carecem de tratamento
- Geno varo não fisiológico: o tratamento é função da gravidade e/ou da evolução clínica:

- Doença de Blount: cirurgia a partir do grau III da doença

- Geno valgo não fisiológico: tratamento em função da gravidade e/ou da evolução clínica:

- Geno valgo idiopático:

- Se sintomático

- Alterações da marcha

- Dificuldade na corrida

- Gonalgia

- Mau alinhamento patelar

- Instabilidade ligamentar

- Preocupação com o aspecto estético

- Geno valgo secundário: a necessidade de tratamento é frequente

Tipos de tratamento

A) Conservador

B) Cirúrgico

\section{A) Tratamento Conservador}

\section{- Isolado}

- Tratamento dos estados carenciais: ex. défice dietético - raquitismo

- Ortóteses

- Fisioterapia

- Associado a tratamento cirúrgico

- Tratamento de raquitismos não carenciais: alterações metabólicas no raquitismo hipofosfatémico vitamina D resistente (de difícil correcção)

- Bifosfonatos (osteogénese imperfeita)

\section{B) Tratamento Cirúrgico}

- Hemiepifisiodese temporária: paragem provisória do crescimento de um dos lados da cartilagem de crescimento (interna ou externa) - cirurgia de modelagem do crescimento ósseo através de grampos ou placas/parafusos ${ }^{7}$

- Hemiepifisiodese definitiva - paragem definitiva do crescimento ósseo por brocagem percutânea da cartilagem de crescimento ou cirurgia invasiva

- Osteotomia correctiva da deformidade

Discussão em relação às opções cirúrgicas:

1) Localização dos grampos /Placas 


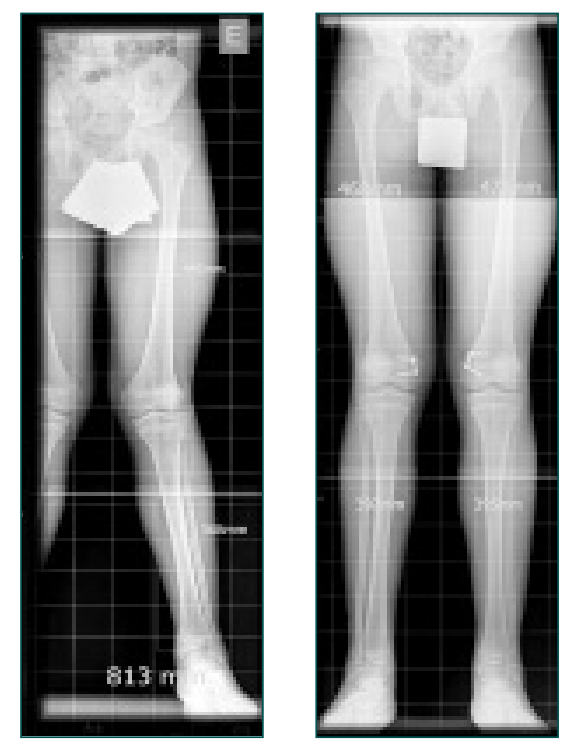

Figura 7. Pré-operatório joelho valgo e pós-operatório após epifisiodese

Na cirurgia de modelagem do crescimento ósseo pretende-se regular a maneira como o osso cresce. Assim, os grampos/placas são colocados no lado externo quando se pretende que o osso cresça mais do lado interno (deformidade em varo) e vice-versa, quando se pretende corrigir um valgo.

\section{2) Indicações de hemi-epifisiodese temporária}

- Doentes sintomáticos, em crescimento

- DIM $>5 \mathrm{~cm}$ depois dos 8 anos de idade

- Eixo de carga fora das zonas normais (sobre a face externa da espinha externa da tíbia).

\section{3) Relação custo-benefício da epifisiodese temporária} As vantagens são que se trata de um método reversível, pouco invasivo e que permite a correcção de desalinhamentos sagitais, evitando as osteotomias realizadas na fase tardia;

As desvantagens são a possível perda da correcção e requerer 2 intervenções cirúrgicas quando se coloca materail de osteossíntese.

\section{4) Relação custo-benefício da epifisiodese definitiva} As vantagens são que não se perde correcção e que se trata de uma cirurgia única;

As desvantagens são que se trata de uma cirurgia

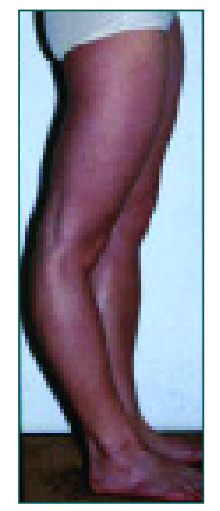

Figura 8. Recurvato à direita, por epifisiodese anterior

permanente, com riscos de provocar eventuais dismetrias (encurtamento do membro operado), sendo difícil decidir qual a altura correcta para a sua realização.

\section{5) Indicações para a osteotomia correctiva}

Trata-se de um método cirúrgico mais agressivo e, por isso, com mais riscos, devendo ser realizada, preferencialmente, no final do crescimento.

\section{Desvios axiais dos membros inferiores} (plano de perfil)

De perfil, observa-se, por vezes, um geno recurvato (joelho em hiper-extensão). A medida deve ser efectuada com um goniómetro, avaliando o ângulo formado pelos eixos anatómicos do fémur e da tíbia (referências anatómicas: saliência do grande trocanter, do côndilo femoral externo e do maléolo externo).

A hiper-extensão do joelho é, habitualmente, constitucional, bilateral e simétrica. Apresenta, geralmente, um máximo de 5 a $10^{\circ}$ e é relativamente frequente (até $15 \%$ da população). Este facto é explicado por uma laxidão ligamentar constitucional (mais frequente nas raparigas). Nalguns casos patológicos, o geno recurvato pode ultrapassar os $30^{\circ}$, como ocorre, por exemplo, após rotura ligamentar posterior, após poliomielite (raramente) ou, ainda, após paragem prematura da cartilagem de crescimento na sua porção anterior (epifisiodese pós-traumática).

Em contrapartida, a existência de um flexo (ligeiro défice de extensão) do joelho é muito mais rara (a não ser em casos patológicos).

Os casos patológicos (recurvatos de mais que $10^{\circ} \mathrm{e}$ 
flexos) deverão ser referenciados à Ortopedia pois obrigam, na maioria das situações, a uma correcção cirúrgica.

\section{CONCLUSÕES}

Os desvios de eixo dos membros inferiores da criança são uma situação comum durante o desenvolvimento, em especial nos primeiros anos de vida.

Na maioria dos casos, trata-se de alterações benignas, correspondendo apenas a desvios do crescimento padrão, sem caracter patológico. É o caso dos joelhos varos, que devem estar corrigidos até aos 2-3 anos e dos joelhos valgus, que se mantêm, por vezes, até aos 8 anos (devendo fazer-se uma avaliação nas consultas dos 2 e dos 8 anos). Estas situações fisiológicas têm tendência a corrigir espontâneamente, razão pela qual não necessitam de qualquer terapêutica nessa fase. O Médico de Família e o Pediatra devem estar atentos aos sinais de alarme, que são fundamentalmente clínicos: uma deformidade congénita, uma assimetria dos membros ou uma deformidade progressiva, fora dos parâmetros correspondentes à idade, que exigem, sempre, uma avaliação por um especialista.

\section{REFERÊNCIAS BIBLIOGRÁFICAS}

1. Herring JA, editor. Tachdjian's Pediatric Orthopaedics. 3rd ed. Philadelphia:W.B. Saunders; 2002.

2. Staheli LT. Fundamentals of Pediatric Orthopedics. 3rd ed. Philadelphia: Lippincott Williams \& Wilkins; 2007.

3. Seabra J. Conceitos básicos de ortopedia infantil. $3^{\mathrm{a}}$ ed. Coimbra: ASIC; 1997.

4. Stevens PM, McWilliams B, Mohr A. Gait analysis of stapling for genu valgum. J Pediatr Orthop 2004 Jan-Feb; 24 (1): 70-4.

5. Stevens PM, Maguire M, Dales MD, Robins AJ. Physeal stapling for idiopathic genu valgum. J Pediatr Orthop 1999 Sep-Oct; 19(5): 645-9.

6. McCarthy JJ, Kim DH, Eilert RE. Posttraumatic genu valgum: operative versus nonoperative treatment. J Pediatr Orthop 1998 Jul-Ago; 18 (4): 518-21.

7. Lerat JL. Orthopédie, Sémiologie, Traumatologie, base de données pour l'enseignement. Paris: Collège Français des Orthopédistes et Traumatologues; 2005.

Os autores declararam não possuir conflitos de interesses

ENDEREÇO PARA CORRESPONDÊNCIA
Manuel Cassiano Neves
Serviço de Ortopedia
Hospital Dona Estefânia
Rua Jacinta Marto
1169-045 Lisboa
Tel: 213126632
E-mail: mcassiano@chlc.min-saude.pt

ENDEREÇO PARA CORRESPONDÊNCIA

Hospital Dona Estefânia

Rua Jacinta Marto

Tel: 213126632

E-mail:mcassiano@chlc.min-saude.pt

\section{ABSTRACT}

\section{AXIAL DEFORMITY OF THE LOWER LIMBS}

Axial deformity of the lower limbs is one of the major causes of referral on a Paediatric Orthopaedic Clinic. This is due to the high percentage of children (20\%) with axial bone deformities in the lower limbs and to the fact that there is a «tradition» according to which these children will require the use of a corrective shoe, at a certain time, in order to treat this type of deformity.

It is therefore of the essence to distinguish the physiologic from the pathologic, to differentiate the different aetiologies, correcting them at an early stage and also to determine when the deformity has a chance of being spontaneously corrected or if, on the other hand, it requires a surgical correction

Keywords: Development; Varus Knee; Valgus Knee; Recurvatum. 